\title{
Irrationality and Transcendence of Alternating Series Via Continued Fractions
}

\author{
Jonathan Sondow
}

\begin{abstract}
Euler gave recipes for converting alternating series of two types, I and II, into equivalent continued fractions, i.e., ones whose convergents equal the partial sums. A condition we prove for irrationality of a continued fraction then allows easy proofs that $e$, sin 1 , and the primorial constant are irrational. Our main result is that, if a series of type II is equivalent to a simple continued fraction, then the sum is transcendental and its irrationality measure exceeds 2 . We construct all $\aleph_{0}^{\aleph_{0}}=\mathfrak{c}$ such series and recover the transcendence of the Davison-Shallit and Cahen constants. Along the way, we mention $\pi$, the golden ratio, Fermat, Fibonacci, and Liouville numbers, Sylvester's sequence, Pierce expansions, Mahler's method, Engel series, and theorems of Lambert, Sierpiński, and Thue-Siegel-Roth. We also make three conjectures.
\end{abstract}

1. INTRODUCTIO. In a 1979 lecture on the Life and Work of Leonhard Euler, André Weil suggested "that our students of mathematics would profit much more from a study of Euler's Introductio in Analysin Infinitorum, rather than of the available modern textbooks" [17, p. xii]. The last chapter of the Introductio is "On Continued Fractions." In it, after giving their form, Euler "next look[s] for an equivalent expression in the usual way of expressing fractions" and derives formulas for the convergents. $\mathrm{He}$ then converts a continued fraction into an equivalent alternating series, i.e., one whose partial sums equal the convergents. He "can now consider the converse problem. Given an alternating series, find a continued fraction such that the series representing the value of the continued fraction is the given series."

In Proposition 1 and Theorem 1 , we recall Euler's solutions for alternating series of two types, I and II. Lemma1, a simplification of Nathan's theorem on irrationality of a continued fraction, then yields conditions for irrationality of the sum of a type I or II series. They easily imply the irrationality of $e, \sin 1$, and the shifted-Fermat-number and primorial constants, and give a simple proof of Sierpiński's theorem.

Our main result is that, if a type II series is equivalent to a simple continued fraction, then the sum has irrationality measure greater than 2 , and so must be transcendental, by the Thue-Siegel-Roth theorem on rational approximations to algebraic numbers.

Corollary 1 constructs all such series and shows that their sums form a continuum of distinct transcendental numbers, including the Davison-Shallit constant.

Corollary 2 gives explicitly the simple continued fractions for "naturally-occurring" transcendental numbers in a doubly-infinite family which contains Cahen's constant.

Finally, Proposition 2 provides irrationality and transcendence conditions for families of non-alternating series, including the Kellogg-Curtiss constant. Here the proofs involve partial sums instead of continued fractions.

Along the way, we encounter $\pi$, Fibonacci and golden rectangle numbers, an alternating Liouville constant, Sylvester's sequence, Pierce expansions, Mahler's method, and Engel series. We also make three conjectures; one on $e^{-1}$ is an analog of Sondow's conjecture on $e$, recently proven by Berndt, Kim, and Zaharescu.

The rest of the paper is organized as follows. Lemma 1 and Proposition 1 are in Section 2, Theorem 1, Corollary 1, and Conjectures 1 and 2 are in Section 3, Corollary 2 is in Section 4, and Proposition 2 and Conjecture 3 are in Section 5. 
2. CONTINUED FRACTIONS AND IRRATIONALITY. In 1761 Lambert [26] derived a continued fraction for $\tan x$ and showed that its value is irrational for rational $x \neq 0$. Since $\tan \frac{\pi}{4}=1$ is rational, Lambert had established that $\pi$ is irrational. For modern treatments of his proof, see [15, §3.6] and [25].

Let us denote the positive integers by $\mathbb{N}$ and the rational numbers by $\mathbb{Q}$. Lemma 1 provides a sufficient condition for irrationality of the value of a continued fraction with all elements in $\mathbb{N}$. (Lambert's has both positive and negative elements.) The statement and quick proof are simplifications of Nathan's theorem in [28].

Lemma 1 (Irrationality Lemma). Let $\alpha$ be the value of a continued fraction

$$
\alpha=\frac{b_{1}}{a_{1}+\frac{b_{2}}{a_{2}+\cdot \cdot}}
$$

where $a_{n} \in \mathbb{N}$ and $b_{n} \in \mathbb{N}$ and $a_{n} \geq b_{n}$ for $n=1,2,3, \ldots$ Then $\alpha \notin \mathbb{Q}$.

Proof. If $\alpha \in \mathbb{Q}$, define the $n$th "tail" of $\alpha$ to be the value of the continued fraction

$$
\alpha_{n}:=\frac{b_{n+1}}{a_{n+1}+\frac{b_{n+2}}{a_{n+2}+\cdot \cdot}}, \quad \text { so } \quad \alpha_{n}=\frac{b_{n+1}}{a_{n+1}+\alpha_{n+1}},
$$

for all $n \geq 0$. The hypotheses ensure that $0<\alpha_{n}<1$ for all $n \geq 0$. As $\alpha_{0}=\alpha$, and $\alpha_{n} \in \mathbb{Q}$ implies $\alpha_{n+1} \in \mathbb{Q}$, we can write $\alpha_{n}=u_{n} / v_{n}$, where $u_{n}$ and $v_{n}$ are coprime positive integers with $u_{n}<v_{n}$. Thus from (1) we get

$$
\frac{u_{n+1}}{v_{n+1}}=\alpha_{n+1}=\frac{v_{n} b_{n+1}-u_{n} a_{n+1}}{u_{n}},
$$

so $u_{n+1}<v_{n+1} \leq u_{n}$. But then $\left(u_{n}\right)_{n \geq 0}$ is a strictly decreasing, infinite sequence of positive integers, which is impossible. Therefore, $\alpha \notin \mathbb{Q}$.

For instance, if $a_{n}=1$ and $b_{n}=1$ for all $n$, then by Lemma 1

$$
\alpha=\frac{1}{1+\frac{1}{1+\cdot \cdot}}=\frac{1}{1+\alpha}>0 \quad \Longrightarrow \quad \alpha=\frac{\sqrt{5}-1}{2} \notin \mathbb{Q} .
$$

Thus the golden ratio $\varphi:=\alpha^{-1}$ is irrational. For more on $\varphi$, see Examples 2 and 6 ,

Lemma1 1 generalizes the irrationality of an infinite simple continued fraction, i.e., one with all partial numerators $b_{n}=1$ and all partial quotients (or partial denominators) $a_{n} \in \mathbb{N}$.

Our hypothesis $a_{n} \geq b_{n}$ is weaker than Nathan's $a_{n}>b_{n}$. Ours is also sharp: with the even weaker hypothesis $a_{n} \geq b_{n}-1$, the lemma would be false, e.g.,

$$
\alpha=\frac{2}{1+\frac{2}{1+\cdot \cdot}}=\frac{2}{1+\alpha}>0 \quad \Longrightarrow \quad \alpha=1 \in \mathbb{Q} .
$$

Lemma 1 holds more generally when $a_{n} \geq b_{n}$ for all sufficiently large $n$. There is also a condition for irrationality of a continued fraction with both positive and negative 
integers $a_{n}$ and $b_{n}$, namely, that $\left|a_{n}\right| \geq\left|b_{n}\right|+1$; see, e.g., [15, §3.6]. We have chosen simplicity over generality here and elsewhere in the paper.

We now apply the Irrationality Lemma to our first kind of alternating series, type I.

Proposition 1. Let $B_{0}<B_{1}<B_{2}<\cdots$ be positive integers.

(i). Then there is an equivalence

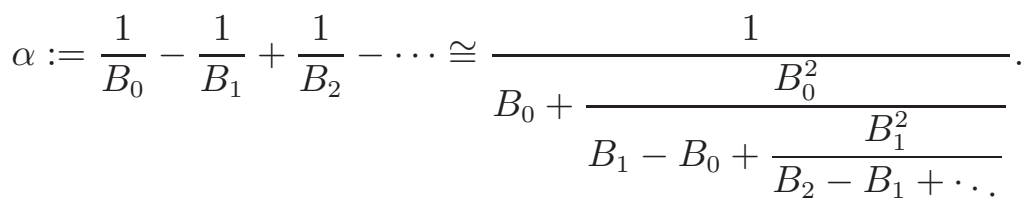

(ii). Suppose that

$$
B_{n+1} \geq B_{n}\left(B_{n}+1\right) \text { for all } n \geq 0 .
$$

Then the sum $\alpha$ is irrational.

Proof. (i). Euler establishes the equivalence in [17, §369]; for example,

$$
\frac{1}{B_{0}}-\frac{1}{B_{1}}=\frac{1}{B_{0}+\frac{B_{0}^{2}}{B_{1}-B_{0}}}
$$

(ii). Set $a_{1}=B_{0}, b_{1}=1, a_{n+1}=B_{n}-B_{n-1}$, and $b_{n+1}=B_{n-1}^{2}$ for $n \geq 1$. Then (3) guarantees that $a_{n} \geq b_{n}$ for all $n$, so by Lemma 1 the value of the continued fraction in (2) is irrational. By (i), that value equals the sum $\alpha$, so $\alpha \notin \mathbb{Q}$.

Proposition 1 provides an easy proof of Sierpinski's theorem, which states that, if (3) holds with all $B_{n} \in \mathbb{N}$, then $\alpha:=\sum_{n=0}^{\infty}(-1)^{n} B_{n}^{-1} \notin \mathbb{Q}$. Sierpiński [34] (see also Cahen [9]) showed moreover that such a representation of any irrational number $\alpha$ in $(0,1)$ exists and is unique. For extensions of his theorem, see Badea [2], Duverney [13], and Nyblom [29].

Note that part (ii) and Sierpinski's theorem are sharp: if $B_{n+1}+1=B_{n}\left(B_{n}+1\right)$ for all $n \geq 0$, then $\left(B_{n+1}+1\right)^{-1}=B_{n}^{-1}-\left(B_{n}+1\right)^{-1}$, so by telescoping

$$
\sum_{n=0}^{\infty} \frac{(-1)^{n}}{B_{n}}=\sum_{n=0}^{\infty}\left(\frac{(-1)^{n}}{B_{n}+1}+\frac{(-1)^{n}}{B_{n+1}+1}\right)=\frac{1}{B_{0}+1} \in \mathbb{Q} .
$$

Example 1. The Fermat numbers $F_{n}=2^{2^{n}}+1$ form the sequence [35, A000215]

$$
\left(F_{n}\right)_{n \geq 0}=3,5,17,257,65537,4294967297,18446744073709551617, \ldots .
$$

Let us define the shifted-Fermat-number constant $F$ to be the alternating sum of reciprocals of the numbers $F_{n}-2$ (for them, see [35, A051179])

$$
F:=\sum_{n=0}^{\infty} \frac{(-1)^{n}}{F_{n}-2}=\sum_{n=0}^{\infty} \frac{(-1)^{n}}{2^{2^{n}}-1}=1-\frac{1}{3}+\frac{1}{15}-\frac{1}{255}+\cdots=0.7294270 \ldots
$$

The numbers $B_{n}:=2^{2^{n}}-1$ satisfy (3), so the shifted-Fermat-number constant $F$ is irrational. For a generalization with a different proof, take $\epsilon=-1$ in [13, Corollary 3.3]. We return to $F$ in Example 5 .

The next section studies irrationality and transcendence of our second kind of alternating series, type II, which is a special case of type I. 
3. SIMPLE CONTINUED FRACTIONS AND TRANSCENDENCE. Our main results are Theorem 1 and Corollaries 1 and 2 We denote the algebraic numbers by $\mathbb{A}$ (others denote them by $\overline{\mathbb{Q}}$, the algebraic closure of $\mathbb{Q}$ ).

Theorem 1. Fix positive integers $A_{0}, A_{1}, A_{2}, \ldots$, with $A_{n} \geq 2$ for all $n \geq 1$.

(i). For any positive real numbers $x_{0}, x_{1}, x_{2}, \ldots$, we have the equivalence between an alternating series and a continued fraction

$$
\alpha:=\sum_{n=0}^{\infty} \frac{(-1)^{n}}{A_{0} A_{1} \cdots A_{n}} \cong \frac{x_{0}}{A_{0} x_{0}+\frac{A_{0} x_{0} x_{1}}{\left(A_{1}-1\right) x_{1}+\frac{A_{1} x_{1} x_{2}}{\left(A_{2}-1\right) x_{2}+\cdot \cdot}}} .
$$

(ii). If $A_{n+1}>A_{n}$ for all $n \geq 0$, then $\alpha$ is irrational.

(iii). If the continued fraction is simple for some $x_{0}, x_{1}, x_{2}, \ldots$, then $\alpha$ is a transcendental number, with irrationality measure $\mu(\alpha) \geq 2.5$.

The irrationality measure (or irrationality exponent) $\mu(\rho)$ of a real number $\rho$ is defined as (see [3, 4, 7], [8, §1.4], [15, Chapter 9], [18, §2.22], [36])

$$
\mu(\rho):=\sup \left\{\mu>0: 0<\left|\rho-\frac{p}{q}\right|<\frac{1}{q^{\mu}} \text { for infinitely many } \frac{p}{q} \in \mathbb{Q}\right\} .
$$

By the famous Thue-Siegel-Roth theorem [1], [8, p. 22], [15, p. 147], [18, p. 172], [22, p. 176]

$$
\mu(\rho) \begin{cases}=1 & \text { if } \rho \text { is rational, } \\ =2 & \text { if } \rho \text { is irrational, but algebraic } \\ \geq 2 & \text { if } \rho \text { is transcendental. }\end{cases}
$$

Proof of Theorem 1 (i). Apply Proposition 11 part (i), with $B_{n}:=A_{0} A_{1} \cdots A_{n}$ for $n \geq 0$. Since $B_{n}-B_{n-1}=\left(A_{n}-1\right) A_{0} A_{1} \cdots A_{n-1}$, cancelling the common factors $A_{0}, A_{0} A_{1}, A_{0} A_{1} A_{2}, \ldots$ in the resulting continued fraction gives

$$
\begin{aligned}
& \frac{1}{A_{0}}-\frac{1}{A_{0} A_{1}}+\frac{1}{A_{0} A_{1} A_{2}}-\cdots \cong \frac{1}{A_{0}+\frac{A_{b}^{\text {p }}}{\left(A_{1}-1\right) A_{0}+\frac{A_{0}^{\text {d }} A_{1}^{2}}{\left(A_{2}-1\right) A_{0} A_{1}+\cdot \cdot}}} \\
& \cong \frac{1}{A_{0}+\frac{A_{0}}{A_{1}-1+\frac{A_{0} A_{1}^{\text {q }}}{\left(A_{2}-1\right) A_{0} A_{1}+\cdot \cdot}}} \cong \frac{1}{A_{0}+\frac{A_{0}}{A_{1}-1+\frac{A_{1}}{A_{2}-1+\cdot \cdot}}},
\end{aligned}
$$

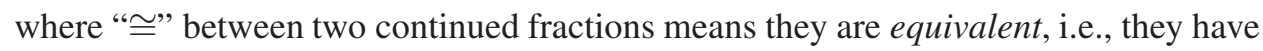
the same convergents (see [15, p. 25]; for two numerical continued fractions which are equivalent but not equal, see Example 4 below). This proves the special case of (i) in which all $x_{n}=1$ (compare to [17, $\left.\S 370\right]$ ). The general case follows by cancelling the common factors $x_{0}, x_{1}, x_{2}, \ldots$ in (4).

(ii). In Lemma11 we take $a_{1}:=A_{0}, b_{1}:=1, a_{n}:=A_{n-1}-1$, and $b_{n}:=A_{n-2}$ for $n \geq 2$. Then $A_{n+1}>A_{n}$ implies $a_{n} \geq b_{n}$ for all $n \geq 1$, so $\alpha \notin \mathbb{Q}$. 
(iii). (Compare to the proof of [12, Theorem 3].) Redefining $a_{1}, a_{2}, \ldots$, we write the simple continued fraction for $\alpha$, and its $n$th convergent, as usual as

$$
\alpha=0+\frac{1}{a_{1}+\frac{1}{a_{2}+\cdot \cdot}}=\left[0, a_{1}, a_{2}, \ldots\right] \quad \text { and } \quad \frac{p_{n}}{q_{n}}=\left[0, a_{1}, a_{2}, \ldots, a_{n}\right]
$$

The hypothesis in (iii) means that

$$
\sum_{i=0}^{n} \frac{(-1)^{i}}{A_{0} A_{1} \cdots A_{i}}=\frac{p_{n+1}}{q_{n+1}} \text { for } n \geq 0 .
$$

A classical theorem [22, Theorem 150] and relation (6) imply, respectively, that

$$
\frac{(-1)^{n}}{q_{n} q_{n+1}}=\frac{p_{n+1}}{q_{n+1}}-\frac{p_{n}}{q_{n}}=\frac{(-1)^{n}}{A_{0} A_{1} \cdots A_{n}}
$$

for $n \geq 1$. Hence $q_{n} q_{n+1}=A_{0} A_{1} \cdots A_{n}$; since $q_{0}=1$ and $q_{1}=A_{0}$, this also holds for $n=0$. It follows that the divisibility $q_{n} q_{n+1} \mid q_{n+1} q_{n+2}$ holds; hence $q_{n} \mid q_{n+2}$. A standard identity [22, Theorem 149] is

$$
q_{n+2}=a_{n+2} q_{n+1}+q_{n},
$$

so $q_{n} \mid a_{n+2} q_{n+1}$. Multiplying (7) by $q_{n} q_{n+1}$, we deduce that $\operatorname{gcd}\left(q_{n}, q_{n+1}\right)=1$, so $q_{n} \mid a_{n+2}$. Define $w_{0}, w_{1}, \ldots$ in $\mathbb{N}$ by $w_{0}=a_{1}$ and $w_{n+1} q_{n}=a_{n+2}$ for $n \geq 0$. By a "simple lemma" [12, Lemma 2],

$$
w_{n} q_{n-1} \geq \sqrt{q_{n}} \text { for infinitely many } n .
$$

Now, from (6), a classical inequality [8, p. 24], the equality $a_{n+1}=w_{n} q_{n-1}$, and (9), respectively, we see that

$$
0<\left|\alpha-\frac{p_{n}}{q_{n}}\right|<\frac{1}{a_{n+1} q_{n}^{2}}=\frac{1}{w_{n} q_{n-1} q_{n}^{2}} \leq \frac{1}{q_{n}^{5 / 2}}
$$

infinitely often. This and definition (5) imply $\mu(\alpha) \geq 2.5$. By the Thue-Siegel-Roth theorem, $\mu(\rho) \leq 2$ if $\rho \in \mathbb{A}$, so $\alpha \notin \mathbb{A}$. This completes the proof of Theorem 1

Note that the hypothesis in (ii) is sharp: if $A_{n}=A_{0}>1$ for all $n>0$, then the series in (4) is geometric, with sum $\alpha=\left(A_{0}+1\right)^{-1} \in \mathbb{Q}$. Also, in (ii) the inequality $A_{n+1}>A_{n}$ is much weaker than that in (3) with $B_{n}=A_{0} A_{1} \cdots A_{n}$, which amounts to $A_{n+1}>A_{0} A_{1} \cdots A_{n}$. Compare Examples 1 and 5

For any strictly increasing sequence of positive integers $A_{0}<A_{1}<A_{2}<\cdots$, finite or infinite, the alternating sum

$$
\alpha:=\frac{1}{A_{0}}-\frac{1}{A_{0} A_{1}}+\frac{1}{A_{0} A_{1} A_{2}}-\cdots
$$

is called the Pierce expansion of $\alpha$. Any number $\alpha \in(0,1)$ has a unique Pierce expansion, which is infinite if, and only if, $\alpha$ is irrational [30, 31, 32, 34]. The "only if" part follows immediately from (ii). 
Example 2. The Pierce expansion of $\varphi^{-1}$ begins [35, A118242, A006276]

$$
\frac{1}{\varphi}=\frac{1}{1}-\frac{1}{1 \cdot 2}+\frac{1}{1 \cdot 2 \cdot 4}-\frac{1}{1 \cdot 2 \cdot 4 \cdot 17}+\frac{1}{1 \cdot 2 \cdot 4 \cdot 17 \cdot 19}-\cdots .
$$

As $\varphi^{-1} \in \mathbb{A}$, we see that the hypothesis in (iii) cannot be omitted. Combined with the next example, this shows that, if the Pierce expansion of $\alpha \notin \mathbb{Q}$ is not equivalent to a simple continued fraction, then $\alpha \in \mathbb{A}$ is possible, but so is $\alpha \notin \mathbb{A}$.

Example 3. Euler [17, p. 325] says, "Something especially deserving of our attention is the number $e \ldots$. " The Taylor series $e^{t}=\sum_{n=0}^{\infty} t^{n} n !^{-1}$ and (i) lead to the Pierce expansion of $e^{-1}$ and the equivalence

$$
e^{-1}=\sum_{n=2}^{\infty} \frac{(-1)^{n}}{2 \cdot 3 \cdot 4 \cdots n} \cong \frac{x_{0}}{2 x_{0}+\frac{2 x_{0} x_{1}}{2 x_{1}+\frac{3 x_{1} x_{2}}{3 x_{2}+\frac{4 x_{2} x_{3}}{4 x_{3}+\cdot \cdot}}} .}
$$

Part (ii) now gives an easy proof that $e$ is irrational. The Taylor series for $\sin t$ and $\cos t$ lead to similar proofs that $\sin \frac{1}{k}$ and $\cos \frac{1}{k}$ are irrational for all $k \in \mathbb{N}$.

From (10) we also see that a strong converse to (iii) is not true. Namely, although $e^{-1} \notin \mathbb{A}$ (because $e \notin \mathbb{A}$ by Hermite [15, §12.14]), the type II series for $e^{-1}$ in (10) is not equivalent to a simple continued fraction. Indeed, when $x_{0}, x_{1}, \ldots$ are chosen so that all partial numerators in the continued fraction for $e^{-1}$ in (10) equal 1

$$
\frac{1}{e}=\frac{1}{2+\frac{2(1 / 2)}{2(1 / 2)+\frac{3(1 / 2)(2 / 3)}{3(2 / 3)+\frac{4(2 / 3)(3 / 8)}{4(3 / 8)+\cdot \cdot}}}}=\frac{1}{2+\frac{1}{1+\frac{1}{2+\frac{1}{(3 / 2)+\cdot \cdot}}}}
$$

the partial quotients do not all lie in $\mathbb{N}$. For a weaker converse to (iii), which is also not true, see Example 8

By (11), the simple continued fraction for $e^{-1}$ begins $e^{-1}=[0,2,1,2, \ldots]$. From 110) (or by inspection), the first four convergents are also partial sums of the Taylor series $e^{-1}=\sum_{n=0}^{\infty}(-1)^{n} n !^{-1}$.

Conjecture 1. Only four partial sums of the Taylor series for $e^{-1}$ are convergents to $e^{-1}$, namely, $0,1 / 2,1 / 3$, and $3 / 8$.

Conjecture 1 is an analog for $e^{-1}$ of the fact that only two partial sums of the Taylor series for e are convergents to e, namely, 2 and $8 / 3$. This property of $e$ was conjectured by Sondow [36], partially proven by him and Schalm [37], and recently proven in full by Berndt, Kim, and Zaharescu [6].

Example 4. An analog of series (10) for $e^{-1}$, with the factorial $n$ ! replaced by the primorial $p_{n} \#$, is "the constant obtained through Pierce retro-expansion of the prime sequence" [35, A132120], which we dub the primorial constant

$$
P:=\sum_{n=1}^{\infty} \frac{(-1)^{n-1}}{p_{n} \#}=\frac{1}{2}-\frac{1}{2 \cdot 3}+\frac{1}{2 \cdot 3 \cdot 5}-\frac{1}{2 \cdot 3 \cdot 5 \cdot 7}+\frac{1}{2 \cdot 3 \cdot 5 \cdot 7 \cdot 11}-\cdots
$$




$$
=\frac{1}{2}-\frac{1}{6}+\frac{1}{30}-\frac{1}{210}+\frac{1}{2310}-\cdots=0.3623062223 \ldots .
$$

Proposition 1, part (i), and Theorem 1, parts (i) and (ii), imply that

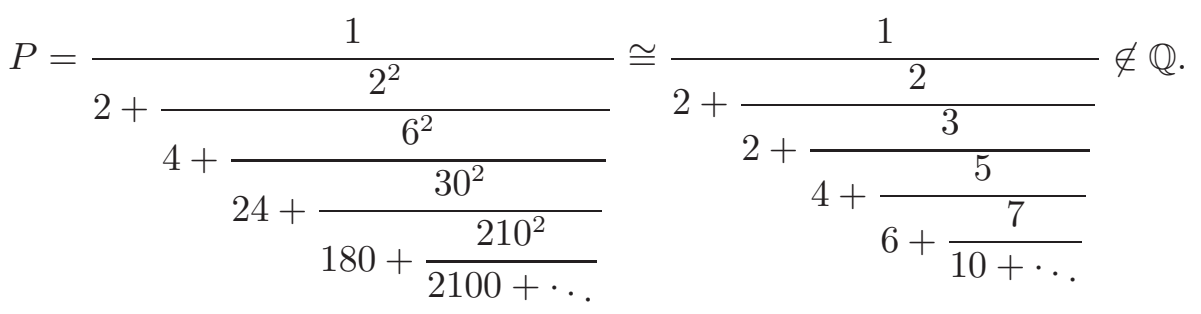

Conjecture 2. The primorial constant $P$ is transcendental.

Example 5. By induction, for $n \geq 0$ the shifted Fermat number $F_{n}-2$ can be factored as the product of all smaller Fermat numbers

$$
F_{n}-2=2^{2^{n}}-1=\prod_{k=0}^{n-1}\left(2^{2^{k}}+1\right)=F_{0} F_{1} \cdots F_{n-1},
$$

where the empty product equals 1 when $n=0$. (From (12) Pólya deduced that $F_{0}, F_{1}, F_{2}, \ldots$ are pairwise coprime, thereby giving an alternate proof to Euclid's theorem on the infinitude of the primes [22, §2.4].) The constant $F$ in Example 1 thus has Pierce expansion

$$
F=\sum_{n=0}^{\infty} \frac{(-1)^{n}}{F_{0} F_{1} \cdots F_{n-1}}=\frac{1}{1}-\frac{1}{1 \cdot 3}+\frac{1}{1 \cdot 3 \cdot 5}-\frac{1}{1 \cdot 3 \cdot 5 \cdot 17}+\cdots
$$

Part (ii) of Theorem 1 now gives a second proof that $F \notin \mathbb{Q}$. Moreover, parts (i) of Proposition 1 and Theorem 1 yield the equivalent continued fractions

$$
F=\frac{1}{1+\frac{1^{2}}{2+\frac{3^{2}}{12+\frac{15^{2}}{240+\cdot \cdot}}}} \cong \frac{1}{1+\frac{1}{2+\frac{3}{4+\frac{5}{16+\cdot \cdot}}}}
$$

Theorem 1 does not yield $F \notin \mathbb{A}$, but Duverney [16] has proven it by other methods.

Remark 1. Non-alternating series involving $F_{n}$ have also been studied. In 1963, Golomb [21] proved that the sum $G:=\sum_{n=0}^{\infty} F_{n}^{-1}$ is irrational. Two years later, Mahler [27] remarked that $G$ is in fact transcendental, as a consequence of a general theorem he proved in 1929—see [14, pp. 194-195]. (Mahler's method [15, §12.3] proves the transcendence of values, at certain algebraic points, of functions that satisfy a type of functional equation.) Recently, Coons [10] showed that $G$ has irrationality measure $\mu(G)=2$. In the pre-Mahler year 1916, Kempner [24] proved that the number $\kappa:=\sum_{n=0}^{\infty}\left(F_{n}-1\right)^{-1}=\sum_{n=0}^{\infty} 2^{-2^{n}}$ is transcendental; see Adamczewski [1] for five proofs with interesting comments. (The second proof applies Mahler's method to the function $f(x):=\sum_{n=0}^{\infty} x^{2^{n}}$, which is defined when $|x|<1$, satisfies the functional equation $f\left(x^{2}\right)=f(x)-x$, and has the value $f(1 / 2)=\kappa$.) 
The next example shows that the sufficient condition for transcendence of the sum of a type II series in Theorem $\square$ does not extend to the more general type I series in Proposition 1.

Example 6. Let $\left(f_{n}\right)_{n \geq 0}=1,1,2,3,5,8,13, \ldots$ be the positive Fibonacci numbers [35. A000045], defined by $f_{0}=1, f_{1}=1$, and $f_{n+1}=f_{n}+f_{n-1}$ for $n \geq 1$. The product $B_{n}:=f_{n} f_{n+1}$ is a golden rectangle number [35, A001654]. The difference between successive golden rectangle numbers is a square:

$$
B_{n}-B_{n-1}=f_{n} f_{n+1}-f_{n-1} f_{n}=f_{n}\left(f_{n+1}-f_{n-1}\right)=f_{n}^{2} .
$$

Therefore, using Proposition 1, part (i), and cancelling common factors $f_{1}^{2}, f_{2}^{2}, \ldots$, we obtain the equivalence

$$
\alpha:=\sum_{n=0}^{\infty} \frac{(-1)^{n}}{f_{n} f_{n+1}} \cong \frac{1}{f_{0} f_{1}+\frac{f_{0}^{2} f_{1}^{\mathcal{X}}}{f_{1}^{\mathcal{Z}}+\frac{f_{1}^{\mathcal{X} f_{2}^{2}}}{f_{2}^{Z}+\cdot \cdot}}}=[0,1,1,1, \ldots] .
$$

The latter is the simple continued fraction expansion of $\alpha=\varphi^{-1} \in \mathbb{A}$. This shows that, given $B_{0}<B_{1}<B_{2}<\cdots$ in $\mathbb{N}$, the sum of the series $\alpha:=\sum_{n=0}^{\infty}(-1)^{n} B_{n}^{-1}$ might not be transcendental, even if the series is equivalent to a simple continued fraction. (However, if in addition $B_{n-1}$ divides $B_{n}$ for all $n \geq 1$, then $\alpha \notin \mathbb{A}$, by Theorem 1 with $A_{0}:=B_{0}$ and $A_{n}:=B_{n} / B_{n-1}$ for $n \geq 1$.)

Remark 2. Example 6 is a special case of the following well-known fact. For any irrational number $\rho$ with simple continued fraction expansion $\rho=\left[a_{0}, a_{1}, a_{2}, \ldots\right]$ and nth convergent $p_{n} / q_{n}$, there is an equivalence

$$
\rho=a_{0}+\sum_{n=0}^{\infty} \frac{(-1)^{n}}{q_{n} q_{n+1}} \cong\left[a_{0}, a_{1}, a_{2}, \ldots\right] .
$$

(Proof. Replacing $\rho$ with $\rho-a_{0}$, we may assume that $a_{0}=0$. Note that $q_{0}=1$. Setting $B_{n}=q_{n} q_{n+1}$, we use (8) to get $B_{n}-B_{n-1}=a_{n+1} q_{n}^{2}$, generalizing relation (13). The rest of the proof is like the argument in Example 6, and is omitted.)

By part (i) of Theorem 11 if the continued fraction in (4) is simple for some $x_{0}, x_{1}, \ldots$, then the series in (4) is equivalent to a simple continued fraction, i.e., (6) holds. Conversely, it is not hard to show by induction that, if (6) holds, then the continued fraction in (4) is simple for some $x_{0}, x_{1}, \ldots$. For instance, if the partial sums $A_{0}^{-1}$ and $A_{0}^{-1}-\left(A_{0} A_{1}\right)^{-1}$ equal the convergents $a_{1}^{-1}$ and $\left(a_{1}+a_{2}^{-1}\right)^{-1}$, respectively, then $A_{0}=a_{1}$ and $\left(A_{1}-1\right) A_{0}^{-1}=a_{2} \in \mathbb{N}$, so the choices $x_{0}=1$ and $x_{1}=A_{0}^{-1}$ give the finite simple continued fraction

$$
\frac{x_{0}}{A_{0} x_{0}+\frac{A_{0} x_{0} x_{1}}{\left(A_{1}-1\right) x_{1}}}=\frac{1}{A_{0}+\frac{1}{\left(A_{1}-1\right) A_{0}^{-1}}}=\left[0, a_{1}, a_{2}\right] .
$$

We now give a method for constructing all examples of Theorem 1 part (iii). 
Corollary 1. (i). Construct a sequence of positive integers $\left(A_{n}\right)_{n \geq 0}$ in three steps. Step 1. Choose a sequence $\left(M_{n}\right)_{n \geq 0}$ with all $M_{n} \in \mathbb{N}$.

Step 2. Let $\left(N_{n}\right)_{n \geq 1}$ satisfy the recursion

$$
N_{1}=1, N_{2}=M_{0} \text {, and } N_{n+2}=\left(M_{n} N_{n+1}+1\right) N_{n} \text { for } n \geq 1 \text {. }
$$

Step 3. Define $\left(A_{n}\right)_{n \geq 0}$ by

$$
A_{0}=M_{0} \text { and } A_{n}=M_{n} N_{n+1}+1 \text { for } n \geq 1 .
$$

Then there exists $\left(x_{n}\right)_{n \geq 0}$ such that (4) is an equivalence between an alternating series and a simple continued fraction, namely,

$$
\alpha:=\sum_{n=0}^{\infty} \frac{(-1)^{n}}{A_{0} A_{1} \cdots A_{n}} \cong\left[0, M_{0}, M_{1} N_{1}, M_{2} N_{2}, M_{3} N_{3}, \ldots\right] .
$$

(ii). Conversely, if the continued fraction in (4) is simple for some $\left(x_{n}\right)_{n \geq 0}$, then the sequence $\left(A_{n}\right)_{n \geq 0}$ in (4) can be constructed by Steps 1, 2, 3.

(iii). The series in (16) is the Pierce expansion of $\alpha$, that is, $A_{n+1}>A_{n}$ for $n \geq 0$.

(iv). Distinct sequences $\left(M_{n}\right)_{n \geq 0} \neq\left(M_{n}^{\prime}\right)_{n \geq 0}$ in Step 1 lead to distinct transcendental numbers $\alpha \neq \alpha^{\prime}$ in (16). In particular, if $\mathbb{S}$ denotes the set of real numbers $\alpha$ whose Pierce expansion is equivalent to a simple continued fraction, then $\# \mathbb{S}=\aleph_{0}^{\aleph_{0}}=\mathfrak{c}$.

Proof. By definition, the continued fraction in (4) is simple if, and only if,

$$
\begin{aligned}
& \text { (a). } x_{0}=1, \\
& \text { (b). } A_{n} x_{n} x_{n+1}=1 \text { for } n \geq 0, \\
& \text { (c). } A_{0} x_{0} \in \mathbb{N} \text {, and } \\
& \text { (d). }\left(A_{n}-1\right) x_{n} \in \mathbb{N} \text { for } n \geq 1 \text {. }
\end{aligned}
$$

(i). Set $x_{0}=1$ and $x_{n}=N_{n} / N_{n+1}$ for $n \geq 1$. From formulas (15) and 14 we get $A_{n}=N_{n+2} / N_{n}$ for $n \geq 1$. It is now easy to verify (a), (b), (c), and (d). Observing that $\left(A_{n}-1\right) x_{n}=M_{n} N_{n}$ for $n \geq 1$, the equivalence (4) gives (16). This proves (i). (ii). Assume (a), (b), (c), and (d). Then $A_{n} \in \mathbb{N}$ implies that $x_{n} \in \mathbb{Q}$ for $n \geq 1$, so $x_{n}=N_{n} / D_{n}$, where $N_{n} \in \mathbb{N}$ and $D_{n} \in \mathbb{N}$, with $\operatorname{gcd}\left(N_{n}, D_{n}\right)=1$. From (a) and (b), we get $N_{1}=1$ and $D_{1}=A_{0}$. From (d), we see that $D_{n} \mid\left(A_{n}-1\right)$ for $n \geq 1$, so there exists $M_{n} \in \mathbb{N}$ such that $A_{n}=M_{n} D_{n}+1$. Since (b) implies $A_{n} N_{n} N_{n+1}=D_{n} D_{n+1}$, we get

$$
\left(M_{n} D_{n}+1\right) N_{n} N_{n+1}=D_{n} D_{n+1} \text { for } n \geq 1 .
$$

Consequently, $N_{n+1} \mid D_{n} D_{n+1}$, so $N_{n+1} \mid D_{n}$. Also, $D_{n} \mid\left(M_{n} D_{n}+1\right) N_{n} N_{n+1}$, so $D_{n} \mid N_{n+1}$. Thus $D_{n}=N_{n+1}$ for all $n \geq 1$; in particular, $N_{2}=D_{1}=A_{0}$. Making replacements in (17) and in $A_{n}=M_{n} D_{n}+1$, we obtain (14) and (15), respectively. This proves (ii).

(iii). Note that (14) and (15) give $A_{n+1}=M_{n+1} A_{n} N_{n}+1>A_{n}$ for $n \geq 0$.

(iv). By Theorem 1 the sum $\alpha$ is transcendental. It now suffices to show that, given $\alpha=\left[0, M_{0}, M_{1} N_{1}, M_{2} N_{2}, \ldots\right]$ and $\alpha^{\prime}=\left[0, M_{0}^{\prime}, M_{1}^{\prime} N_{1}^{\prime}, M_{2}^{\prime} N_{2}^{\prime}, \ldots\right]$, if $\alpha=\alpha^{\prime}$, then $M_{n}=M_{n}^{\prime}$ for all $n \geq 0$. By the uniqueness of simple continued fraction expansion, $M_{0}=M_{0}^{\prime}$ and $M_{k} N_{k}=M_{k}^{\prime} N_{k}^{\prime}$ for $k \geq 1$. Using (14), the rest of the proof is an easy induction, which we omit. This completes the proof of the corollary. 
Example 7. Choosing the constant sequence $M_{n}=1$ yields $N_{1}=1, N_{2}=1$, and $N_{n+2}=\left(N_{n+1}+1\right) N_{n}$ for $n \geq 1$. Then $A_{0}=1$ and $A_{n}=N_{n+1}+1$ for $n \geq 1$, so

$$
A_{n}=1,2,3,4,9,28,225,6076,1361025, \ldots
$$

(see [35, A007704]). By (iv), we recover the transcendence of the Davison-Shallit constant [12, Example A] (see also [18, pp. 436, 445], [35, A242724])

$$
D:=\sum_{n=0}^{\infty} \frac{(-1)^{n}}{A_{0} A_{1} \cdots A_{n}}=1-\frac{1}{2}+\frac{1}{6}-\frac{1}{24}+\frac{1}{216}-\cdots=0.62946502045 \ldots
$$

and, by (i), the expansion [12, p. 122], [35, A006277]

$$
D=\left[0,1, N_{1}, N_{2}, N_{3}, \ldots\right]=[0,1,1,1,2,3,8,27,224,6075,1361024, \ldots] .
$$

Example 8. Let us define an alternating Liouville constant by the series

$$
\begin{aligned}
\lambda:=\sum_{n=2}^{\infty} \frac{(-1)^{n}}{10^{n !}} & =\frac{1}{10^{2}}-\frac{1}{10^{6}}+\frac{1}{10^{24}}-\frac{1}{10^{120}}+\cdots \\
& =0.009999000000000000000000 \underbrace{99 \ldots 9}_{96} 00 \ldots .
\end{aligned}
$$

For $n=1,2,3, \ldots$, the $n$th partial sum of the series satisfies

$$
\frac{P_{n}}{Q_{n}}:=\sum_{k=2}^{n+1} \frac{(-1)^{k}}{10^{k !}} \Longrightarrow 0<\left|\lambda-\frac{P_{n}}{Q_{n}}\right|<\frac{1}{10^{(n+2) !}}=\frac{1}{Q_{n}^{n+2}} .
$$

From this and (5), we infer that $\lambda$ has irrationality measure $\mu(\lambda)=\infty$. By definition, $\lambda$ is therefore a Liouville number, so Liouville's theorem [8, §1.4], [15, §9.3], [22, $\S 11.7]$ (or its descendant, the Thue-Siegel-Roth theorem) implies $\lambda$ is transcendental.

On the other hand, its Pierce expansion

$$
\lambda=\sum_{n=0}^{\infty} \frac{(-1)^{n}}{A_{0} A_{1} \cdots A_{n}}=\frac{1}{10^{2 !}}-\frac{1}{10^{2 !} 10^{3 !-2 !}}+\frac{1}{10^{2 !} 10^{3 !-2 !} 10^{4 !-3 !}}-\cdots
$$

cannot be constructed from any sequence $\left(M_{n}\right)_{n>0}$ as in (i). (Proof. If it could, then $M_{0}=A_{0}=10^{2 !}$ would imply $M_{1} 10^{2 !}+1=A_{1}=10^{3 !-2 !}$, contradicting $M_{1} \in \mathbb{N}$.)

Hence by (ii) a converse to Theorem 1 part (iii), weaker than the false converse in Example 3, is also not true. Namely, although $\lambda \notin \mathbb{A}$ and $\mu(\lambda) \geq 2.5$, the type II series for $\lambda$ in 18 is not equivalent to a simple continued fraction.

More positively, one can show that, if a sequence $\left(M_{n}\right)_{n \geq 0}$ in (i) grows sufficiently rapidly, then the sum $\alpha$ in the equivalence (16) is a Liouville number.

The next section gives further applications of Theorem 1

4. SYLVESTER'S SEQUENCE AND CAHEN'S CONSTANT. There are not many "naturally-occurring" transcendental numbers for which the simple continued fraction is known explicitly. They include the beautiful expansions 


$$
\begin{aligned}
e-1 & =[1,1,2,1,1,4,1,1,6,1,1,8,1,1,10,1,1,12,1,1,14, \ldots], \\
\tan 1 & =[1,1,1,3,1,5,1,7,1,9,1,11,1,13,1,15,1,17,1,19, \ldots], \\
1 / \tanh 1 & =[1,3,5,7,9,11,13,15,17,19,21,23,25,27,29,31,33, \ldots], \\
I_{0}(2) / I_{1}(2) & =[1,2,3,4,5,6,7,8,9,10,11,12,13,14,15,16,17,18, \ldots],
\end{aligned}
$$

and those of $e^{2 / q}, \tan \frac{1}{q}, \tanh \frac{1}{q}$, and $I_{\frac{p}{q}}\left(\frac{2}{q}\right) / I_{1+\frac{p}{q}}\left(\frac{2}{q}\right)$, for $p$ and $q$ in $\mathbb{N}$, where $I_{c}(x)$ is a modified, or hyperbolic, Bessel function of the first kind [15, Chapter 3]. References to several others are given in $[\mathbf{1 2}, \S \mathrm{V}]$.

Theorem 1 yields a doubly-infinite family of such numbers. We define them by a natural recursion, independently of Corollary 1 .

Corollary 2. Fix $k \in \mathbb{N}$ and $\ell \in \mathbb{N}$. For $n \geq 0$, define $s_{n}=s_{n}(k, \ell)$ by the recurrence

$$
s_{0}=k \text { and } s_{n}=\left(s_{0} s_{1} \cdots s_{n-1}\right)^{\ell}+1 \text { for } n \geq 1 .
$$

(i). Then there is an equivalence

$$
C_{k, \ell}:=\sum_{n=0}^{\infty} \frac{(-1)^{n}}{s_{n+1}-1} \cong\left[a_{0}, a_{1}, a_{2}, \ldots\right],
$$

where the partial quotients of the simple continued fraction are

$$
a_{0}=0, a_{1}=s_{0}^{\ell}, \text { and } a_{n+1}=\left(s_{n}^{\ell}-1\right) \prod_{i=0}^{n-1}\left(s_{i}^{\ell}\right)^{(-1)^{n+i}} \in \mathbb{N} \text { for } n \geq 1 .
$$

(ii). The sum $C_{k, \ell}$ is transcendental, and $C_{k, \ell}=C_{k^{\prime}, \ell^{\prime}}$ only when $(k, \ell)=\left(k^{\prime}, \ell^{\prime}\right)$.

(iii). The double-exponential lower bound $a_{n}>\left(k^{\ell}+1\right)^{(\ell+1)^{n-4}}$ holds for all $n \geq 4$. (iv). There are the summations

$$
\sum_{n=0}^{\infty} \frac{s_{n}^{\ell}-1}{s_{n+1}-1}=1 \quad \text { and } \quad \sum_{n=0}^{\infty} \frac{s_{2 n+1}^{\ell}-1}{s_{2 n+2}-1}=C_{k, \ell} .
$$

(v). Taking $\ell=1$ gives

$$
\begin{aligned}
C_{k, 1} & =\frac{1}{s_{0}}-\frac{1}{s_{0} s_{1}}+\frac{1}{s_{0} s_{1} s_{2}}-\frac{1}{s_{0} s_{1} s_{2} s_{3}}+\frac{1}{s_{0} s_{1} s_{2} s_{3} s_{4}}-\frac{1}{s_{0} s_{1} s_{2} s_{3} s_{4} s_{5}}+\cdots \\
& \cong\left[0, s_{0}, 1,\left(s_{0}\right)^{2},\left(s_{1}\right)^{2},\left(s_{0} s_{2}\right)^{2},\left(s_{1} s_{3}\right)^{2},\left(s_{0} s_{2} s_{4}\right)^{2},\left(s_{1} s_{3} s_{5}\right)^{2}, \ldots\right] .
\end{aligned}
$$

(vi). For odd $n \geq 1$ and even $m \geq 2$, the partial quotients $a_{n}$ and $a_{m}$ of $C_{k, 1}$ are coprime.

Proof. (i). Set $A_{n}:=s_{n}^{\ell}$ for $n \geq 0$. Then (19) gives $s_{n+1}-1=A_{0} A_{1} \cdots A_{n}$, so by Theorem 1 for any $x_{0}, x_{1}, \ldots$ in $\mathbb{R}^{+}$there is an equivalence

$$
C_{k, \ell}=\sum_{n=0}^{\infty} \frac{(-1)^{n}}{s_{0}^{\ell} s_{1}^{\ell} \cdots s_{n}^{\ell}} \cong \frac{x_{0}}{s_{0}^{\ell} x_{0} x_{1}} x_{0}+\frac{s_{1}^{\ell} x_{1} x_{2}}{\left(s_{1}^{\ell}-1\right) x_{1}+\frac{1}{\left(s_{2}^{\ell}-1\right) x_{2}+\cdot \cdot}} .
$$


The partial numerators equal 1 when $x_{0}=1$ and $x_{n+1}=\left(s_{n}^{\ell} x_{n}\right)^{-1}$ for $n \geq 0$. By induction, the solution of this recursion is

$$
x_{n}=\prod_{i=0}^{n-1}\left(s_{i}^{\ell}\right)^{(-1)^{n+i}} \text { for } n \geq 1 .
$$

The partial quotients are then $a_{0}=0, a_{1}=s_{0}^{\ell} x_{0}=s_{0}^{\ell}$, and $a_{n+1}=\left(s_{n}^{\ell}-1\right) x_{n}$ for $n \geq 1$. Substituting $s_{n}^{\ell}-1=\left(s_{0}^{\ell} s_{1}^{\ell} \cdots s_{n-1}^{\ell}+1\right)^{\ell}-1$ and expanding the binomial, the 1 s cancel, so $s_{0}^{\ell} s_{1}^{\ell} \cdots s_{n-1}^{\ell}$ divides $s_{n}^{\ell}-1$ and $a_{n+1} \in \mathbb{N}$. This proves (i).

(ii). Theorem 1 and (i) imply $C_{k, \ell} \notin \mathbb{A}$. From $a_{1}=k^{\ell}$ and $a_{2}=\left(\left(k^{\ell}+1\right)^{\ell}-1\right) k^{-\ell}$, we deduce that $C_{k, \ell} \neq C_{k^{\prime}, \ell^{\prime}}$ when $(k, \ell) \neq\left(k^{\prime}, \ell^{\prime}\right)$. This proves (ii).

(iii). Let $\alpha_{n}:=s_{n}-1$. Then (19) implies $\alpha_{n+1}=\alpha_{n}\left(\alpha_{n}+1\right)^{\ell}>\alpha_{n}^{\ell+1}$ for $n \geq 1$. As $\alpha_{2}=k^{\ell}\left(k^{\ell}+1\right)^{\ell} \geq k^{\ell}+1$, induction yields $\alpha_{n} \geq\left(k^{\ell}+1\right)^{(\ell+1)^{n-2}}$ for $n \geq 2$. Since (i) implies $a_{n} \geq s_{n-3}^{2 \ell}>\alpha_{n-3}^{2 \ell} \geq \alpha_{n-3}^{\ell+1}$, we get (iii).

(iv). For $n>0$, definition (19) implies $s_{n+1}-1=\left(s_{n}-1\right) s_{n}^{\ell}$, so

$$
\frac{1}{s_{n}-1}-\frac{1}{s_{n+1}-1}=\frac{s_{n}^{\ell}-1}{s_{n+1}-1} \text { for } n \geq 1 \text {. }
$$

Hence the first series in (iv) telescopes to $\left(s_{0}^{\ell}-1\right)\left(s_{1}-1\right)^{-1}+\left(s_{1}-1\right)^{-1}=1$. Replacing $n$ with $2 n+1$ in (20), we sum from $n=0$ to $\infty$ and obtain the second equality in (iv).

(v). Set $\ell=1$ in parts (i) and (ii).

(vi). Recursion (19) yields $\operatorname{gcd}\left(s_{i}, s_{j}\right)=1$ for $i \neq j$, so (ii) follows from (i). This completes the proof of the corollary.

Example 9. Take $(k, \ell)=(1,1)$. Sylvester's sequence [39, 40] is defined as

$$
\left(S_{n}\right)_{n \geq 0}:=\left(s_{n+1}(1,1)\right)_{n \geq 0}=2,3,7,43,1807,3263443,10650056950807, \ldots
$$

(see [12, p. 123], [18, pp. 436, 444], [20], [35, A000058]). Sylvester's sequence satisfies the recursion $S_{0}=2$ and $S_{n+1}=\left(S_{n}-1\right) S_{n}+1$ for $n \geq 0$.

Likewise, $C:=C_{1,1}$ defines Cahen's constant [9], [18, §6.7], [35, A118227]

$$
\begin{aligned}
C & =\sum_{n=0}^{\infty} \frac{(-1)^{n}}{S_{n}-1}=1-\frac{1}{2}+\frac{1}{6}-\frac{1}{42}+\frac{1}{1806}-\cdots=0.643410546288338 \ldots \\
& =1-\sum_{n=1}^{\infty} \frac{(-1)^{n-1}}{S_{0} S_{1} \cdots S_{n-1}}=1-\frac{1}{2}+\frac{1}{2 \cdot 3}-\frac{1}{2 \cdot 3 \cdot 7}+\frac{1}{2 \cdot 3 \cdot 7 \cdot 43}-\cdots
\end{aligned}
$$

Corollary 2]recovers $C \notin \mathbb{A}$ from [12] and gives the expansion [35, A006279]

$$
\begin{aligned}
C & =\left[0,1,1,1,\left(S_{0}\right)^{2},\left(S_{1}\right)^{2},\left(S_{0} S_{2}\right)^{2},\left(S_{1} S_{3}\right)^{2},\left(S_{0} S_{2} S_{4}\right)^{2},\left(S_{1} S_{3} S_{5}\right)^{2}, \ldots\right] \\
& =\left[0,1,1,1,2^{2}, 3^{2}, 14^{2}, 129^{2}, 25298^{2}, 420984147^{2}, \ldots\right] .
\end{aligned}
$$

Since $\alpha_{n}:=S_{n}-1$ satisfies $\alpha_{n+1}-\alpha_{n}=\alpha_{n}^{2}$ and $\sum_{n=0}^{\infty}(-1)^{n} \alpha_{n}^{-1}=C$, Proposi- 
tion 1 and Theorem 1 give, respectively, the continued fractions

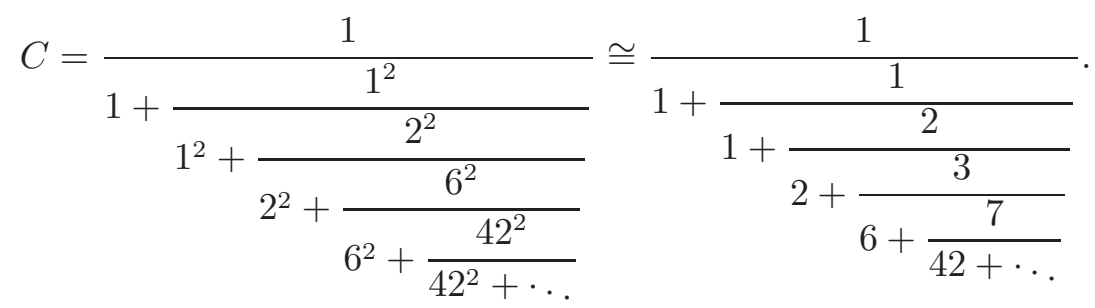

In his 1891 paper "A remark on an expansion of numbers which has some similarities with continued fractions" [9], Cahen defined $C$ and showed that it is irrational. Exactly 100 years later, as an example of their "self-similar" (or "self-generating" [18 $\S 6.7],[19, \S 6.7])$ simple continued fractions, Davison and Shallit [12] proved that $C$ is transcendental and that $C=\left[0,1, q_{0}^{2}, q_{1}^{2}, q_{2}^{2}, \ldots\right]$. (This expansion agrees with (21), by (8) and induction.) For generalizations of [12], see Becker [5] and Töpfer [41].

Example 10. Corollary 2 shows that the Cahen-type constant $C_{k, 1}=[0, k, 1, \ldots]$, so

$$
1>C_{1,1}>\frac{1}{2}>C_{2,1}>\frac{1}{3}>C_{3,1}>\frac{1}{4}>C_{4,1}>\cdots .
$$

When $k=2$ we have $s_{0}(2,1)=2=s_{1}(1,1)=S_{0}$. It follows that in general $s_{n+1}(2,1)=s_{n+2}(1,1)=S_{n+1}$, so

$$
C_{2,1}=\sum_{n=0}^{\infty} \frac{(-1)^{n}}{s_{n+1}(2,1)-1}=\sum_{n=0}^{\infty} \frac{(-1)^{n}}{S_{n+1}-1}=1-\sum_{n=0}^{\infty} \frac{(-1)^{n}}{S_{n}-1}=1-C .
$$

By Corollary 2 ,

$$
C_{2,1}=\left[0,2,1,2^{2}, 3^{2}, 14^{2}, 129^{2}, 25298^{2}, 420984147^{2}, \ldots\right] \notin \mathbb{A} .
$$

Example 11. For an example with $\ell>1$, we take $(k, \ell)=(1,2)$ to get

$$
\left(s_{n+1}(1,2)\right)_{n \geq 0}=2,5,101,1020101,1061522231810040101, \ldots
$$

(see [33] and [35, A231830]). Then $C_{1,2}$ is the transcendental number

$$
\begin{aligned}
C_{1,2} & =1-\frac{1}{2^{2}}+\frac{1}{2^{2} \cdot 5^{2}}-\frac{1}{2^{2} \cdot 5^{2} \cdot 101^{2}}+\cdots \\
& =1-\frac{1}{4}+\frac{1}{100}-\frac{1}{1020100}+\cdots=0.759999019703 \ldots
\end{aligned}
$$

Here $\alpha_{n}:=s_{n+1}(1,2)-1$ satisfies $\alpha_{n+1}-\alpha_{n}=\alpha_{n}^{2}\left(\alpha_{n}+2\right)$, so Proposition 1 Theorem 1 and Corollary 2 give the continued fractions

$$
C_{1,2}=\frac{1}{1+\frac{1^{2}}{1^{2} \cdot 3+\frac{4^{2}}{4^{2} \cdot 6+\frac{100^{2}}{100^{2} \cdot 102+\cdot \cdot}}}} \cong \frac{1}{1+\frac{1}{3+\frac{4}{24+\frac{25}{10200+\cdot \cdot}}}}
$$




$$
\begin{aligned}
\cong & {\left[0,1^{2}, 2^{2}-1,\left(5^{2}-1\right) 2^{-2},\left(101^{2}-1\right) 2^{2} 5^{-2},\left(1020101^{2}-1\right) 2^{-2} 5^{2} 101^{-2},\right.} \\
& \left.\left(1061522231810040101^{2}-1\right) 2^{2} 5^{-2} 101^{2} 1020101^{-2}, \ldots\right] \\
= & {[0,1,3,6,1632,637563750,1767398865801083661443214432, \ldots] . }
\end{aligned}
$$

Our final section studies series of positive terms involving Sylvester-type sequences.

5. SOME NON-ALTERNATING SERIES. Another series formed from Sylvester's sequence is the sum of reciprocals. Setting $(k, \ell)=(1,1)$ in 20 , the right-hand side is then $S_{n}^{-1}$, so the series telescopes to

$$
\sum_{n=0}^{\infty} \frac{1}{S_{n}}=\sum_{n=0}^{\infty}\left(\frac{1}{S_{n}-1}-\frac{1}{S_{n+1}-1}\right)=\frac{1}{S_{0}-1}=1,
$$

a rational number. By contrast, the corresponding alternating sum

$$
\sum_{n=0}^{\infty} \frac{(-1)^{n}}{S_{n}}=\sum_{n=0}^{\infty} \frac{(-1)^{n}}{S_{n}-1}-\sum_{n=0}^{\infty} \frac{(-1)^{n}}{S_{n+1}-1}=C-(1-C)=2 C-1
$$

is transcendental, as are the non-alternating sums

$$
\sum_{n=0}^{\infty} \frac{1}{S_{2 n}}=\sum_{n=0}^{\infty}\left(\frac{1}{S_{2 n}-1}-\frac{1}{S_{2 n+1}-1}\right)=C
$$

and $\sum_{n=0}^{\infty} S_{2 n+1}^{-1}=1-C$.

Finch asked, "What can be said about $\sum_{n=0}^{\infty}\left(S_{n}-1\right)^{-1}=1.6910302067 \ldots$ ?" [18, p. 436]. We denote this constant by

$$
K:=\sum_{n=0}^{\infty} \frac{1}{S_{n}-1}=1+\sum_{n=1}^{\infty} \frac{1}{S_{0} S_{1} \cdots S_{n-1}}
$$

and we name it the Kellogg-Curtiss constant, because Kellogg conjectured [23], and Curtiss proved [11], the following bound on solutions to a unit fraction equation:

$$
x_{i} \in \mathbb{N} \text { and } \sum_{i=0}^{n} \frac{1}{x_{i}}=1 \quad \Longrightarrow \quad \max _{0 \leq i \leq n} x_{i} \leq S_{n}-1 .
$$

Remark 3. By (22), one solution of the equation $\sum_{i=0}^{\infty} x_{i}^{-1}=1$ is $x_{i}=S_{i}$. In fact, this is the solution provided by the "greedy Egyptian fraction algorithm"-see Soundararajan [38]. Likewise, the greedy Egyptian fraction expansion of Cahen's constant $C$ is series (23) with $x_{i}=S_{2 i}$.

The following general result shows in particular that $K$ is irrational.

Proposition 2. For $k \in \mathbb{N}$ and $\ell \in \mathbb{N}$, define the Kellogg-Curtiss-type constant

$$
K_{k, \ell}:=\sum_{n=0}^{\infty} \frac{1}{s_{n+1}(k, \ell)-1}
$$

where the Sylvester-type sequence $\left(s_{n}(k, \ell)\right)_{n \geq 0}$ is defined in Corollary 2 
(i). Then $K_{k, \ell} \notin \mathbb{Q}$. In particular, the Kellogg-Curtiss constant $K=K_{1,1}=1+K_{2,1}$ is irrational.

(ii). If $\ell \geq 2$, then $K_{k, \ell}$ is transcendental and $\mu\left(K_{k, \ell}\right) \geq 3$.

We could prove (i) from the fact that, given a non-decreasing sequence of positive integers $A_{0}, A_{1}, \ldots$, the Engel series $\sum_{n=0}^{\infty}\left(A_{0} A_{1} \cdots A_{n}\right)^{-1}$ converges to an irrational number if (and only if) $A_{n}$ tends to infinity with $n$ (see, e.g., [15, §2.2]). Instead, we give a mostly self-contained proof. It uses partial sums instead of continued fractions (compare to Example 8).

Proof of Proposition 2 Let us fix integers $k \geq 1$ and $\ell \geq 1$, and write $s_{n}$ in place of $s_{n}(k, \ell)$. Then for $n \geq 1$, the $n$th partial sum of the series for $K_{k, \ell}$ is, in lowest terms,

$$
\frac{P_{n}}{Q_{n}}:=\sum_{i=0}^{n-1} \frac{1}{s_{i+1}-1}=\sum_{i=0}^{n-1} \frac{1}{s_{0}^{\ell} s_{1}^{\ell} \cdots s_{i}^{\ell}} \quad \Longrightarrow \quad Q_{n}=s_{0}^{\ell} s_{1}^{\ell} \cdots s_{n-1}^{\ell}=s_{n}-1 .
$$

With this value of $Q_{n}$ we see that

$$
\begin{aligned}
0<K_{k, \ell}-\frac{P_{n}}{Q_{n}} & =\sum_{i=n}^{\infty} \frac{1}{s_{0}^{\ell} s_{1}^{\ell} \cdots s_{i}^{\ell}}=\frac{1}{Q_{n}} \sum_{j=0}^{\infty} \frac{1}{s_{n}^{\ell} \cdots s_{n+j}^{\ell}} \\
& <\frac{1}{Q_{n}} \sum_{j=0}^{\infty} \frac{1}{\left(s_{n}^{\ell}\right)^{j+1}}=\frac{1}{Q_{n}} \frac{1}{s_{n}^{\ell}-1} \leq \frac{1}{Q_{n}^{\ell+1}} .
\end{aligned}
$$

(i). If $K_{k, \ell} \in \mathbb{Q}$, say $K_{k, \ell}=P / Q$, then

$$
K_{k, \ell}-\frac{P_{n}}{Q_{n}}=\frac{P}{Q}-\frac{P_{n}}{Q_{n}} \geq \frac{1}{Q Q_{n}}>\frac{1}{Q_{n}^{2}}
$$

for $n$ so large that $Q_{n}>Q$. But $\ell \geq 1$, so (25) contradicts (24). Therefore, $K_{k, \ell} \notin \mathbb{Q}$. (ii). From (24), we infer that $\mu\left(K_{k, \ell}\right) \geq \ell+1$. If $\ell \geq 2$, then $\mu\left(K_{k, \ell}\right) \geq 3$, so by the Thue-Siegel-Roth theorem, $K_{k, \ell} \notin \mathbb{A}$. This completes the proof of the proposition.

By a similar argument (also not using Theorem 1 or continued fractions), $C_{k, \ell} \notin \mathbb{A}$ for $\ell \geq 2$. The case $\ell=1$ though (which includes Cahen's constant $C$ ) would seem to require using Theorem 1 as in the proof of Corollary 2] However, Duverney [16] has found a proof that $C \notin \mathbb{A}$ which is similar to that of Proposition 2, part (ii). He uses relation (23) and the fact that $S_{2 n+2}>\frac{1}{8} S_{2 n}^{4}$, which follows from $S_{n+1}>\frac{1}{2} S_{n}^{2}$.

Duverney has also answered Finch's question by pointing out that, as a special case of a result of Becker [5, p. 186, Remark (ii)], the Kellogg-Curtiss constant $K$ is transcendental.

Conjecture 3. For $k \geq 1$, the Kellogg-Curtiss-type constant $K_{k, 1}$ is transcendental.

ACKNOWLEDGMENTS. I thank Daniel Duverney and Michael Nyblom for (independently) pointing out the series which shows that Sierpiński's theorem is sharp. I am also indebted to Duverney for generalizing my earlier special case of Corollary 1 I thank Steven Finch for comments on the manuscript and for discussions on [12]. Finally, I am grateful to Yohei Tachiya for a proof that $K$ is irrational. 


\section{REFERENCES}

1. B. Adamczewski, The many faces of the Kempner number, J. Integer Seq. 16 (2013) Article 13.2.15.

2. C. Badea, A theorem on irrationality of infinite series and applications, Acta Arith. 63 (1993) 313-323.

3. N. D. Baruah, B. C. Berndt, H. H. Chan, Ramanujan's series for $1 / \pi$ : a survey, Amer. Math. Monthly 116 (2009) 567-587.

4. K. Beanland, J. W. Roberts, C. Stevenson, Modifications of Thomae's function and differentiability, Amer. Math. Monthly 116 (2009) 531-535.

5. P.-G. Becker, Algebraic independence of the values of certain series by Mahler's method, Monatsh. Math. 114 (1992) 183-198.

6. B. C. Berndt, S. Kim, A. Zaharescu, Diophantine approximation of the exponential function and Sondow's conjecture, Adv. Math. 248 (2013) 1298-1331.

7. J. M. Borwein, S. T. Chapman, I prefer pi: a brief history and anthology of articles in the American Mathematical Monthly, Amer. Math. Monthly 122 (2015) 195-216.

8. J. Borwein, A. van der Poorten, J. Shallit, W. Zudilin, Neverending Fractions: An Introduction to Continued Fractions. Cambridge Univ. Press, Cambridge, 2014.

9. E. Cahen, Note sur un développement des quantités numériques, qui presente quelque analogie avec celui des fractions continues, Nouv. Ann. Math. 10 (1891) 508-514; also available at http://archive.numdam.org/ARCHIVE/NAM/NAM_1891_3_10_/NAM_1891_3_10__508_0/NAM_1891_3_10__508_0.pdf

10. M. Coons, On the rational approximation of the sum of the reciprocals of the Fermat numbers, Ramanujan J. 30 (2013) 39-65.

11. D. R. Curtiss, On Kellogg's Diophantine problem, Amer. Math. Monthly 29 (1922) 380-387; also available at http://www.jstor.org/stable/2299023

12. J. L. Davison, J. O. Shallit, Continued fractions for some alternating series, Monatsh. Math. 111 (1991) 119-126.

13. D. Duverney, Irrationality of fast converging series of rational numbers, J. Math. Sci. Univ. Tokyo 8 (2001) $275-316$.

14. — Transcendence of a fast converging series of rational numbers, Math. Proc. Cambridge Philos. Soc. 130 (2001) 193-207.

15. — Number Theory: An Elementary Introduction Through Diophantine Problems. Monographs in Number Theory, Vol. 4. World Scientific, Singapore, 2010.

16. - Transcendence of Cahen's constant and related numbers, preprint, 2015.

17. L. Euler, Introduction to Analysis of the Infinite. English trans. and Introduction by J. D. Blanton. Springer, New York, 1988.

18. S. R. Finch, Mathematical Constants. Encyclopedia of Mathematics and its Applications 94. Cambridge Univ. Press, Cambridge, 2003.

19. — Errata and addenda to Mathematical Constants, e-print (2020), available at http://arxiv.org/abs/2001.00578

20. S. W. Golomb, On certain nonlinear recurring sequences, Amer. Math. Monthly 70 (1963) 403-405.

21. On the sum of the reciprocals of the Fermat numbers and related irrationalities, Canad. J. Math. 15 (1963) 475-478.

22. G. H. Hardy, E. M. Wright, An Introduction to the Theory of Numbers. Fifth edition. Oxford Univ. Press, Oxford, 1979.

23. O. D. Kellogg, On a Diophantine problem, Amer. Math. Monthly 28 (1921) 300-303.

24. A. J. Kempner, On transcendental numbers, Trans. Amer. Math. Soc. 17 (1916) 476- 482.

25. M. Laczkovich, On Lambert's proof of the irrationality of $\pi$, Amer. Math. Monthly 104 (1997) 439-443.

26. J. H. Lambert, Mémoire sur quelques propriétés remarquables des quantités transcendantes circulaires et logarithmiques, 1768, in L. Berggren, J. Borwein, P. Borwein, Pi, a Source Book, Springer-Verlag, New York, 1997, pp. 129-146.

27. K. Mahler, Remarks on a paper by W. Schwarz, J. Number Theory 1 (1969) 512-521 .

28. J. A. Nathan, The irrationality of $e^{x}$ for nonzero rational $x$, Amer. Math. Monthly 105 (1998) 762-763.

29. M. A. Nyblom, An extension of a result of Sierpiński, J. Number Theory 105 (2004) 49-59.

30. J. Paradís, P. Viader, L. Bibiloni, Approximation to quadratic irrationals and their Pierce expansions, Fib. Quart. 36 (1998) 146-153.

31. — A mathematical excursion: from the three-door problem to a Cantor-type set, Amer. Math. Monthly 106 (1999) 241-251.

32. T. A. Pierce, On an algorithm and its use in approximating roots of algebraic equations, Amer. Math. Monthly 36 (1929) 523-525.

33. S. A. Shirali, A family portrait of primes-a case study in discrimination, Math. Mag. 70 (1997) 263-272.

34. W. Sierpiński, Sur un algorithme pour développer les nombres réels en séries rapidement convergentes, Bull. Internat. Acad. Sci. Lettres Cracovie Sér. A (1911) 113-117. 
35. N. J. A. Sloane, The On-Line Encyclopedia of Integer Sequences, available at https://oeis . org

36. J. Sondow, A geometric proof that $e$ is irrational and a new measure of its irrationality, Amer. Math. Monthly 113 (2006) 637-641 [article], 114 (2007) 659 [editor's endnote].

37. J. Sondow, K. Schalm, Which partial sums of the Taylor series for $e$ are convergents to $e$ ? (and a link to the primes 2, 5, 13,37,463). Part II, in Gems in Experimental Mathematics, T. Amdeberhan, L.A. Medina, V.H. Moll, eds. Contemp. Math., Vol. 517. American Mathematical Society, Providence, 2010, 349-363.

38. K. Soundararajan, Approximating 1 from below using $n$ Egyptian fractions, e-print (2005), available at http://arxiv.org/abs/math/0502247

39. J. J. Sylvester, On a point in the theory of vulgar fractions, Amer. J. Math. 3 (1880) 332-335; also available at http://www.jstor.org/stable/2369261

40. Postscript to note on a point in vulgar fractions, Amer. J. Math. 3 (1880) 388-389; also available at http://www.jstor.org/stable/2369265

41. T. Töpfer, On the transcendence and algebraic independence of certain continued fractions, Monatsh. Math. 117 (1994) 255-262.

This manuscript was submitted posthumously.

The author passed away on January 16, 2020. 